\title{
Control of Structural Sound Radiation and Vibration Using Shunt Piezoelectric Materials*
}

\author{
Stanislaw PIETRZKO** and Qibo MAO*** \\ **Empa, Swiss Federal Laboratories for Materials Science and Technology \\ Ueberlandstrasse 129, CH-8600 Duebendorf, Switzerland \\ E-mail: stanislaw.pietrzko@empa.ch \\ ${ }^{* * *}$ School of Aircraft Engineering, Nanchang HangKong University, \\ Nanchang, 330063, P. R. China
}

\begin{abstract}
In this paper, structural sound and vibration control using passive and semi-active shunt piezoelectric damping circuits is presented. A piezoelectric patch with an electrical shunt circuit is bonded to a base structure. When the structure vibrates, the piezoelectric patch strains and transforms the mechanical energy of the structure into electrical energy, which can be effectively dissipated by the shunt circuit. Hence, the shunt circuit acts as a means of extracting mechanical energy from the base structure. Different types of shunt circuits (such as an $R L$ series circuit, an $R L$ parallel circuit and an $R L-C$ circuit) employed in the passive damping arrangement, are analyzed and compared. Using the impedance method, the general modeling of different shunt piezoelectric damping techniques is presented. The piezoelectric shunt circuit can be seen as an additional frequency-dependence damping of the system. One of the primary concerns in shunt damping is to choose the optimal parameters for shunt circuits. In past efforts most of the proposed tuning methods were based on modal properties of the structure. In this study, a design method based on minimization of the sound power of the structure is proposed. The optimal parameters for shunt circuits are obtained using linear quadratic optimal control theory. Numerical simulations are performed for each of these shunts techniques focusing on minimizing radiated sound power from a clamped plate. It is found that the $R L$ series, $R L$ parallel and pulse-switching circuits have basically the same control performance. The $R L-C$ parallel circuit allows us to reduce the value of the inductance $L$ due to the insertion of an external capacitance $C$. However, the control performance will be reduced simultaneously. The pulse-switching circuit is more stable than an $R L$ series circuit with regard to structural stiffness variations. Finally, experimental results are presented using an $R L$ series/parallel shunt circuit, $R L$-C parallel shunt circuit and pulse-switching circuit.
\end{abstract}

Key words: Shunt Damping Circuit, Structural Damping and Sound Power Control

\section{Introduction}

Structural damping is an important means of reducing vibration, noise and fatigue. The design and implementation of shunt piezoelectric damping as applied to structural vibration control has been intensively investigated in the last decade. The concept of shunt piezoelectric damping can be traced back to Hagood and Flotow [1]. They demonstrated that it is possible to add damping to vibrating structures using piezoelectric elements with passive shunt circuits. Piezoelectric elements are employed here to convert the mechanical

DOI: $10.1299 /$ /jsdd.5.752]

Copyright $\odot 2011$ by JSME 
energy of a vibrating structure to electrical energy which can be dissipated through the resistive components of the shunt circuit. The shunt piezoelectric damping technique does not require an external sensor. Hence, no external energy is added and the stability of the system can be guaranteed.

Following in the footsteps of Hagood and Flotow [1], many types of shunt circuits, such as $R L$ parallel [2-5], $R L-C$ parallel [3,6] and the switching shunt circuit [7-12], have been proposed, these typical shunt circuits are shown in Fig. 1. In general, the passive shunt circuit techniques are an effective method of contributing modal damping. However, the main drawback of the passive shunt circuit is that the shunt piezoelectric is very sensitive to tuning errors and variations in the excitation frequency. To overcome this problem, Corr and Clark [8] proposed pulse-switching shunt piezoelectrics for structural damping. In a pulse-switching circuit, the piezoelectric element is connected to an $R L$ shunt circuit, which allows a generated charge to be applied to the piezoelectric element; similar to direct velocity feedback control.

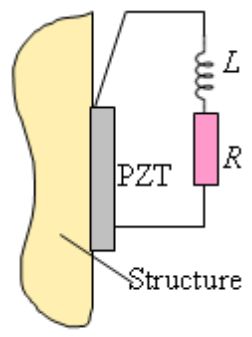

(a)

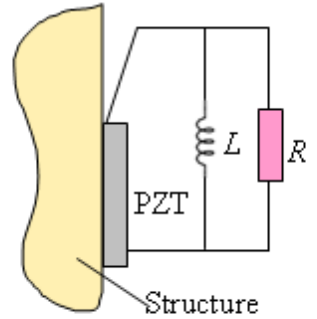

(b)



(c)

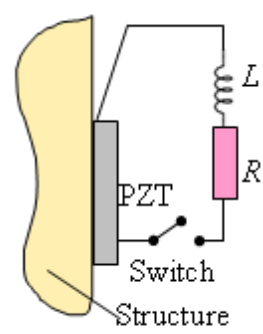

(d)

Fig. 1 Several typical piezoelectric shunt circuits. (a) $R L$ series; (b) $R L$ parallel; (c) $R L-C$ parallel; (d) pulse-switching

In this study, the shunt piezoelectric damping techniques are used to control the sound power radiated from a vibrating structure. The general modelling for the different shunt piezoelectric damping (such as $R L$ series circuit, $R L$ parallel circuit and $R L-C$ circuit) is presented. Then, based on minimizing the sound power of the structure, the optimal parameters for shunt circuits are discussed. The switch-law for the pulse-switching circuit is also discussed in this study, and the detailed numerical calculations are given and discussed. Finally, with the example of a clamped plate, the experimental results are given using $R L$ series/parallel and pulse-switching circuits.

\section{Shunt piezoelectrics}

A piezo-structure is defined to be a structure consisting of embedded or bonded piezoelectric devices. The dynamic modal of the piezo-structure includes the electrical inputs and outputs as well as the modified mass stiffness effect of the structural system due to the additional piezoelectric device. A shunt circuit with a piezoelectric element can be seen as shunt impedance $Z_{s h}$, as shown Fig. 2 .

Using Hamilton's principle, the mechanical and electrical equation of the system can be obtained as follows

$$
\begin{aligned}
& {\left[\boldsymbol{M}_{s}+\boldsymbol{M}_{p z}\right] \ddot{\boldsymbol{\eta}}+\boldsymbol{B} \dot{\boldsymbol{\eta}}+\left[\boldsymbol{K}_{s}+\boldsymbol{K}_{p z}\right] \boldsymbol{\eta}-\operatorname{coup} \cdot V=\boldsymbol{f}} \\
& Q=\operatorname{coup}^{T} \boldsymbol{\eta}+C_{p} V
\end{aligned}
$$

where $\boldsymbol{M}_{s}$ and $\boldsymbol{M}_{p z}$ are mass matrices associated with the structure and piezoceramics, respectively; $\boldsymbol{K}_{s}$ and $\boldsymbol{K}_{p z}$ are stiffness matrices associated with the structure and piezoceramics, respectively; coup is an electromechanical coupling matrix; $\boldsymbol{B}$ is the modal mechanical damping matrix; $\eta$ is the modal coordinate vector; $V$ is the voltage of 
piezoceramics; $Q$ is the charge on the electrical circuit; $\boldsymbol{f}$ is modal force and $C_{p}$ is the capacitance of piezoceramics.

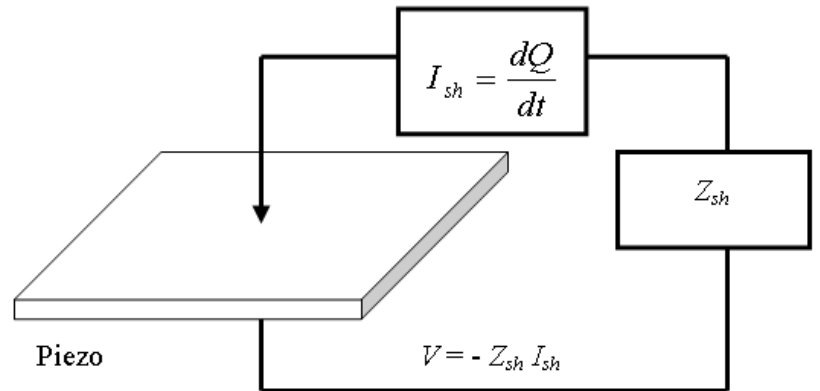

Fig. 2 Feedback current into a PZT due to shunt impedance.

From Fig. 2, we can define the shunt voltage as follows

$$
V=-Z_{s h} I=-Z_{s h} \frac{d Q}{d t}
$$

The charge generated by the PZT patch due to the vibration of the base structure can be determined from Eq. (2). Substituting Eq. (3) into Eq. (2), we get

$$
V=-Z_{s h}\left(\operatorname{coup}^{T} \frac{d \boldsymbol{\eta}}{d t}+C_{p} \frac{d V}{d t}\right)=-Z_{s h}\left(\operatorname{coup}^{T} j \omega \cdot \boldsymbol{\eta}+j \omega C_{p} \cdot V\right)
$$

From Eq. (4), the voltage $V$ can be expressed as

$$
V=-\frac{j \omega Z_{s h} \operatorname{coup}^{T}}{j \omega C_{p} Z_{s h}+1} \eta
$$

Substituting Eq. (5) into Eq. (1), yield

$$
\boldsymbol{M} \ddot{\boldsymbol{\eta}}+\left(\boldsymbol{B}+\boldsymbol{c o u p} \cdot \frac{Z_{s h} \cdot \operatorname{coup} \boldsymbol{p}^{T}}{j \omega C_{p} Z_{s h}+1}\right) \dot{\boldsymbol{\eta}}+\boldsymbol{K} \boldsymbol{\eta}=\boldsymbol{f}
$$

where $\boldsymbol{M}=\boldsymbol{M}_{s}+\boldsymbol{M}_{p z}, \quad \boldsymbol{K}=\boldsymbol{K}_{s}+\boldsymbol{K}_{p z}$.

From Eq. (6), the shunt piezoelectric circuit can be seen as a damper modal, as shown in Fig. 3.

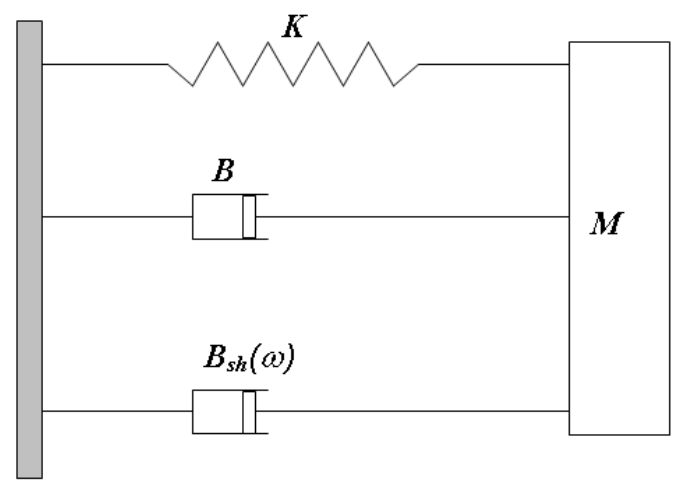

Fig. 3 Frequency dependent damping $\boldsymbol{B}_{s h}(\omega)$ due to shunt piezoelectric device.

From Eq. (6) and Fig. 3, the frequency dependent damping $\boldsymbol{B}_{\boldsymbol{s h}}(\omega)$ can be defined as

$$
B_{s h}(\omega)=\operatorname{coup} \cdot \frac{Z_{s h} \cdot \operatorname{coup}^{T}}{j \omega C_{p} Z_{s h}+1}
$$




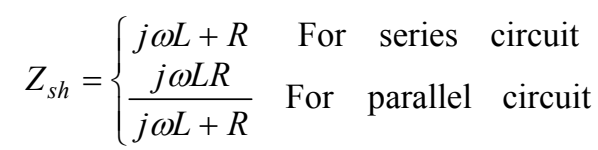

For an $R L-C$ shunt circuit, $B_{s h}(\omega)=\operatorname{coup} \cdot \frac{Z_{s h} \cdot \operatorname{coup}^{T}}{j \omega C_{T} Z_{s h}+1}$

where $Z_{s h}=j \omega L+R$ and $C_{T}=C_{p}+C$

\section{Optimal parameters by minimization of sound power}

Since the shunt piezoelectric circuits are used to minimize the sound power of the vibrating structure, the choice of inductance and resistance is very important.

First, for simplification, the following is defined as:

$$
G\left(Z_{s h}\right)=\frac{1}{C_{p}}+j \omega Z_{s h}, \quad \text { co }=\frac{\text { coup }}{C_{p}}
$$

The modal coordinate vector $\eta$ in Eq. (6) can be obtained and rewritten as:

$$
\boldsymbol{\eta}=\left[\boldsymbol{K}-\omega^{2} \boldsymbol{M}+i \omega \boldsymbol{B}+C_{p} \cdot \boldsymbol{c o} \cdot \boldsymbol{c o} \boldsymbol{o}^{T}-\frac{\boldsymbol{c o} \cdot \boldsymbol{c o}{ }^{T}}{G\left(Z_{s h}\right)}\right]^{-1} \cdot \boldsymbol{f}
$$

As referred to Ozer and Royston's work [6], we now introduce the Sherman Morrison (SM) method for matrix inversion

$$
[\boldsymbol{A}+\boldsymbol{u v}]^{-1}=\boldsymbol{A}^{-1}-\frac{\boldsymbol{A}^{-1} \boldsymbol{u} \boldsymbol{v}^{T} \boldsymbol{A}^{-1}}{1+\boldsymbol{v}^{T} \boldsymbol{A}^{-1} \boldsymbol{u}}
$$

Assume that

$$
\begin{aligned}
& \boldsymbol{A}=\left[\boldsymbol{K}-\omega^{2} \boldsymbol{M}+i \omega \boldsymbol{B}+C_{p} \cdot \boldsymbol{c o} \cdot \boldsymbol{c} \boldsymbol{o}^{T}\right]^{-1} \\
& \boldsymbol{\alpha}=\boldsymbol{A}^{-1}, \quad \boldsymbol{u}=\frac{\boldsymbol{c o}}{G\left(Z_{s h}\right)}, \quad \boldsymbol{v}^{T}=\boldsymbol{c} \boldsymbol{o}^{T}
\end{aligned}
$$

Using Eqs.(11 - 13), Eq. (10) can be rewritten as:

$$
\boldsymbol{\eta}=\left[\boldsymbol{A}+\frac{\boldsymbol{A} \cdot \boldsymbol{c o} \cdot \boldsymbol{c o} \boldsymbol{o}^{T} \cdot \boldsymbol{A}}{G\left(Z_{s h}\right)-\boldsymbol{c o} \boldsymbol{o}^{T} \boldsymbol{A} \cdot \boldsymbol{c o}}\right] \cdot \boldsymbol{f}=\boldsymbol{A} \cdot \boldsymbol{f}+\left[\frac{\boldsymbol{A} \cdot \boldsymbol{c o} \cdot \boldsymbol{c o} \boldsymbol{o}^{T} \cdot \boldsymbol{A}}{G\left(Z_{s h}\right)-\boldsymbol{c o} \boldsymbol{o}^{T} \boldsymbol{A} \cdot \boldsymbol{c o}}\right] \cdot \boldsymbol{f}
$$

where $\boldsymbol{A}=\left[\boldsymbol{K}-\omega^{2} \boldsymbol{M}+i \omega \boldsymbol{B}+C_{p} \cdot \boldsymbol{c o} \cdot \boldsymbol{c o} \boldsymbol{o}^{T}\right]^{-1}$.

From Eq. (14), the modal coordinate vector of the plate with a shunt piezoelectric patch can be rewritten as:

$$
\boldsymbol{\eta}=\boldsymbol{A} \cdot \boldsymbol{f}+F\left(Z_{s h}\right) \cdot\left[\boldsymbol{A} \cdot \boldsymbol{c o} \cdot \boldsymbol{c o}{ }^{T} \cdot \boldsymbol{A}\right] \cdot \boldsymbol{f}=\boldsymbol{\eta}_{p}+F\left(Z_{s h}\right) \cdot \boldsymbol{K}
$$

where $K=\left[\boldsymbol{A} \cdot \boldsymbol{c o} \cdot \boldsymbol{c} \boldsymbol{o}^{T} \cdot \boldsymbol{A}\right] \cdot \boldsymbol{f} ; \quad F\left(Z_{s h}\right)=\frac{1}{G\left(Z_{s h}\right)-\boldsymbol{c o}{ }^{T} \boldsymbol{A} \cdot \boldsymbol{c o}}$, is the coefficient for optimal shunt damping circuits. $\eta_{p}$ is the modal coordinate vector due to the primary source.

The response of the structure is represented by a series of expansion [11]

$$
w(x, y)=\sum_{m=1}^{\infty} \sum_{n=1}^{\infty} \Phi_{m, n}(x, y) \cdot \eta_{m, n}=\boldsymbol{\Phi} \boldsymbol{\eta}
$$

where $\Phi_{m, n}(x, y)$ is the structural mode shape.

Assume that a plate is divided into $J$ elements with equal area. The vector of normal velocities of each of these elements is denoted as $\boldsymbol{v}$. The sound power of the vibrating plate can be calculated using radiation mode approach $[11,13,14]$

$$
W=\boldsymbol{v}^{H} \boldsymbol{R} \boldsymbol{v}
$$

where superscript $H$ denotes the complex conjugate transpose. $\boldsymbol{R}$ is $J \times J$ radiation resistance matrix and the matrix $\boldsymbol{R}$ is real, symmetrical and positive definite.

According to Eqs. (15) and (16), the velocity distribution can be expressed as 


$$
v(x, y)=i \omega w(x, y)=v^{p}(x, y)+K(x, y) \cdot F\left(Z_{s h}\right)
$$

where $\quad \boldsymbol{K}=i \omega \boldsymbol{\Phi}(x, y) \cdot\left[\boldsymbol{A} \cdot \boldsymbol{c o} \cdot \boldsymbol{c} \boldsymbol{o}^{T} \cdot \boldsymbol{A}\right] . \quad v^{p}(x, y)=i \omega \cdot \boldsymbol{\Phi}(x, y) \boldsymbol{A} \cdot \boldsymbol{f}$ is the velocity due to primary source,

Rewrite Eq. (18) into matrix form,

$$
\boldsymbol{v}=\boldsymbol{v}^{p}+\boldsymbol{K} F\left(Z_{s h}\right)
$$

where $\boldsymbol{v}, \boldsymbol{v}^{p}$ and $\boldsymbol{K}$ are $J \times 1$ vectors.

Substituting Eq. (19) into Eq. (17)

$$
\begin{aligned}
& W=\left(\boldsymbol{v}^{p}+\boldsymbol{K} \boldsymbol{F}\right)^{H} \boldsymbol{R}\left(\boldsymbol{v}^{p}+\boldsymbol{K} \boldsymbol{F}\right) \\
& =\boldsymbol{F}^{H} \boldsymbol{K}^{H} \boldsymbol{R} \boldsymbol{K} \boldsymbol{F}+\boldsymbol{F}^{H}\left(\boldsymbol{K}^{H} \boldsymbol{R} \boldsymbol{v}^{p}\right)+\left(\boldsymbol{K}^{H} \boldsymbol{R} \boldsymbol{v}^{p}\right)^{H} \boldsymbol{F}+\left(\boldsymbol{v}^{p}\right)^{H} \boldsymbol{R}\left(\boldsymbol{v}^{p}\right)
\end{aligned}
$$

Clearly the matrix $\boldsymbol{K}^{H} \boldsymbol{R} \boldsymbol{K}$ is positive definite and Eq. (20) is a standard Hermitian quadratic equation, using linear quadratic optimal control theory [15], this equation has a unique global minimum

$$
F_{\text {opt }}=-\left(\boldsymbol{K}^{H} \boldsymbol{R} \boldsymbol{K}\right)^{-1}\left(\boldsymbol{K}^{H} \boldsymbol{R} \boldsymbol{v}^{p}\right)
$$

From Eq. (21), we can obtain the optimal inductance and resistance values for the different shunt piezoelectric circuits.

\section{Switch law for pulse-switching circuit}

In this section, the switch law for pulse-switching circuits is discussed. The model of the circuit is shown in Fig. 1(d). Recall Eqs. (1) and (2), if the switch is shut (meaning turned on)

$$
\boldsymbol{M} \ddot{\boldsymbol{\eta}}+\boldsymbol{B} \dot{\boldsymbol{\eta}}+\boldsymbol{K} \boldsymbol{\eta}-\frac{\text { coup }}{C} Q+\frac{\text { coupcoup }^{T}}{C} \boldsymbol{\eta}=\boldsymbol{f}
$$

The charge produce by the PZT actuator is:

$$
L \ddot{Q}+R \dot{Q}+\frac{1}{C_{p}} Q=\frac{\operatorname{coup}^{T}}{C_{p}} \boldsymbol{\eta}
$$

Assume $\boldsymbol{c o}=\boldsymbol{c o u p} / C_{p}$ and substituting Eq. (23) into Eq. (22) yields

$$
\boldsymbol{M} \ddot{\boldsymbol{\eta}}+\boldsymbol{B} \dot{\boldsymbol{\eta}}+\left[\boldsymbol{K}+C_{p} \cdot \boldsymbol{c o} \cdot \boldsymbol{c o} \boldsymbol{o}^{T}-\frac{\boldsymbol{c o} \cdot \boldsymbol{c o}}{\frac{1}{C_{p}}-\omega^{2} L+i \omega R}\right] \boldsymbol{\eta}=\boldsymbol{f}
$$

It is assumed that the charge applied on the PZT actuator remains constant while the switch is open. The equations for the open-state can be written as

$$
\boldsymbol{M} \ddot{\boldsymbol{\eta}}+\left[\boldsymbol{B}+\frac{-\boldsymbol{c o} \cdot \boldsymbol{c o}}{\frac{1}{C_{p}}-\omega^{2} L+i \omega R} \frac{\boldsymbol{\eta}_{\boldsymbol{0}}}{\dot{\boldsymbol{\eta}}}\right] \dot{\boldsymbol{\eta}}+\left[\boldsymbol{K}+C_{p} \cdot \boldsymbol{c o} \cdot \boldsymbol{c o}{ }^{T}\right] \boldsymbol{\eta}=\boldsymbol{f}
$$

where $\eta_{0}$ is the modal displacement at the time the shunt switch is opened

The fundamental issue regarding a switching circuit is determining when to switch. From Eq.(25), to add maximum damping to the structure, if and only if

$$
\boldsymbol{\eta}_{0} \cdot \operatorname{sign}(\dot{\boldsymbol{\eta}}) \leq 0 \quad \text { and } \quad \boldsymbol{\eta}_{0}=\max |\boldsymbol{\eta}| \quad \text { (When switch-off) }
$$

The natural frequency of the electric circuit is $\omega_{s h}=1 / \sqrt{L C_{p}}$. Assume that the inductance $L$ is quite small and $\omega_{s h} \gg \omega_{0}, \eta_{0}$ in Eq.(25) can be expressed as 


$$
\boldsymbol{\eta}_{0}=\frac{\frac{1}{C_{p}}}{-\omega L^{2}+j \omega R+\frac{1}{C_{p}}} \boldsymbol{\eta}_{\text {switch }-o n}
$$

from Eq.(27), it is found that $\eta_{0}$ reaches the first maximum at switch time

$$
\Delta T_{s h}=\pi \sqrt{L C_{p}}=1 / 2 \text { of the period of the electric circuit }
$$

According to Eqs. $(25-28)$, we can obtain the switch law for pulse-switching circuit.

\section{Numerical calculation}

Assume that a piezoelectric element $(20 \mathrm{~mm} \times 20 \mathrm{~mm})$ is bonded on a clamped plate with size $205 \mathrm{~mm} \times 205 \mathrm{~mm} \times 1 \mathrm{~mm}$. The density and Young's modulus of the plate are $1550 \mathrm{~kg} / \mathrm{m}^{3}$ and $2.75 \times 10^{10} \mathrm{~N} / \mathrm{m}^{2}$, respectively. Assume that the damping ratio of each structural mode is $0.5 \%$. The plate is excited by uniform incident plane waves, so only the (odd, odd) modes can be excited. The center of the PZT is located at $(30 \mathrm{~mm}, 30 \mathrm{~mm})$. The radiated sound power of the plate before and after circuit activation for different shunt circuits is calculated to check the control performance of the different shunt circuits. Fig. 4 shows the control performances of the $R L$ series and $R L$ parallel circuits. It can be found that the $R L$ series and $R L$ parallel circuits have the same control performance.

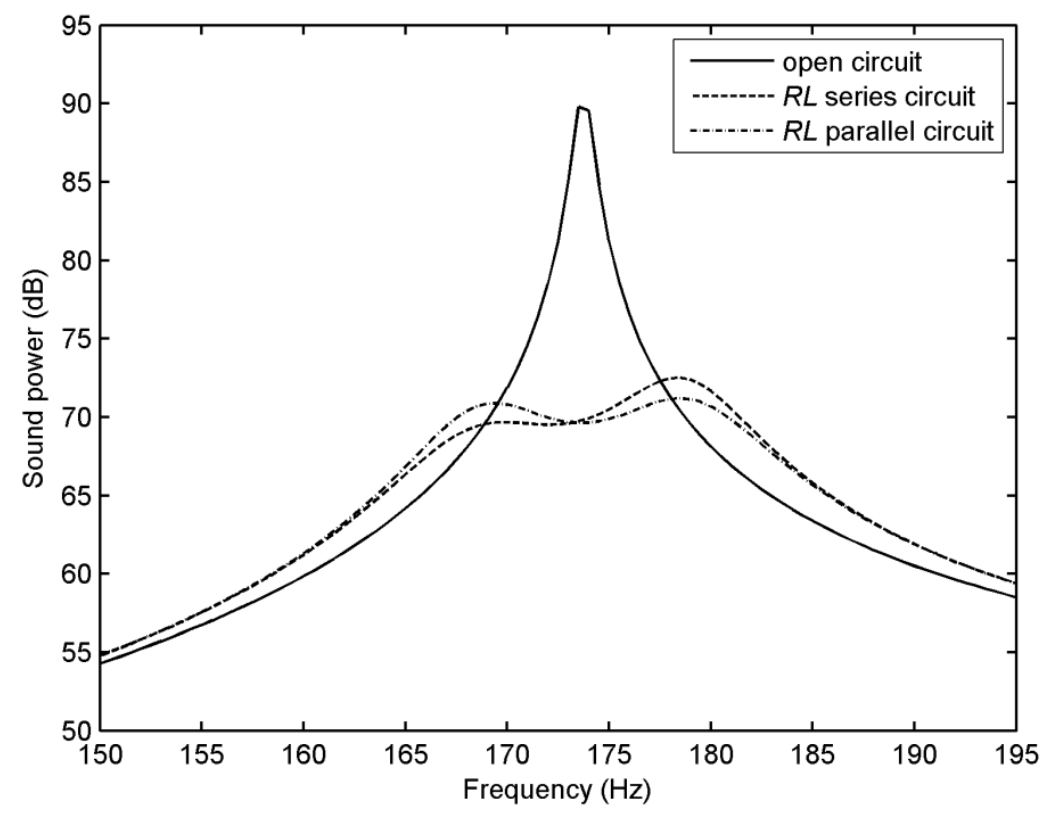

Fig. 4 The control performance of $R L$ series and $R L$ parallel circuit.

The control performance of the $R L-C$ parallel circuit deeply depends on the additional capacitance $C$, as shown in Fig. 5. The value of inductance can be reduced by placing an additional capacitance across the terminals of the piezoelectric element. Inductance and resistance are reduced by the same factor in which capacitance is increased. However, this additional capacitance can also reduce the control performance of shunt circuit. The situation can be viewed as a trade-off between desired component reduction and tolerable performance loss. 


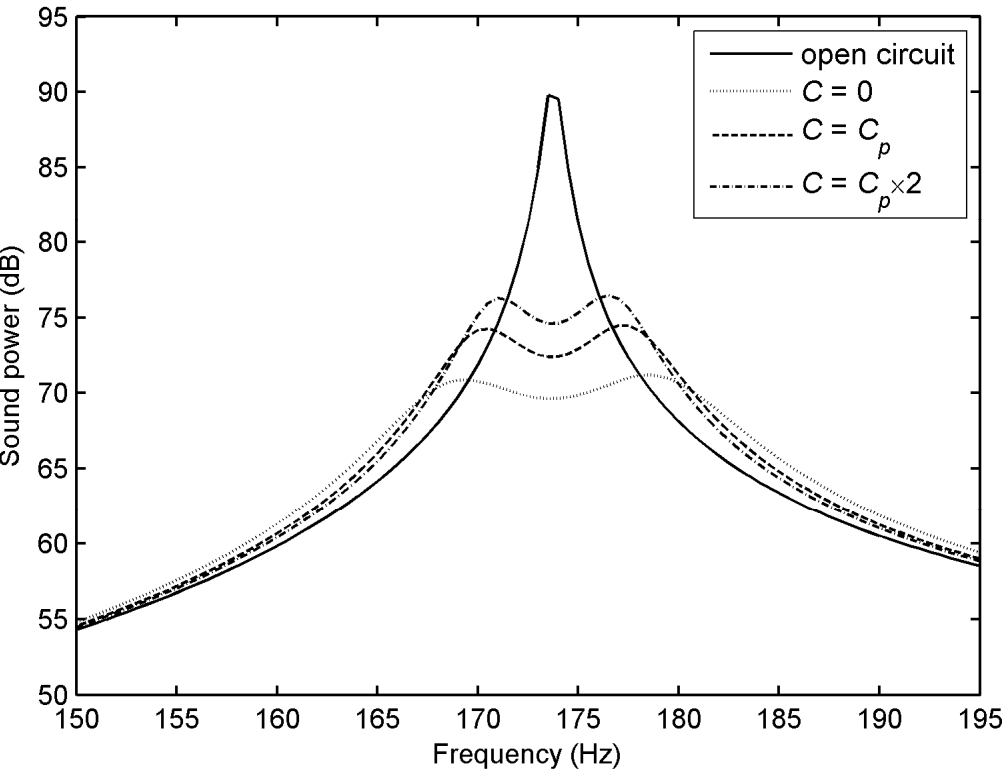

Fig. 5 The control performance of $R L-C$ parallel circuit.

Next, we discuss the control performance of the pulse-switching circuit. For a switching shunt circuit, a fundamental question considered here is when to switch. To answer this question, we show the calculated results in the time-domain. Figs. 6 and 7 show the time response of the velocity and charge for $R L$ series and pulse-switching shunt circuits. It is found that the control performances of the $R L$ series and pulse-switching circuits are almost the same. It should be noted that the optimal inductance required is $4.17 \mathrm{H}$; however, it is only $208 \mathrm{mH}$ for a pulse-switching circuit. Fig. 8 compares the time response for different circuits.

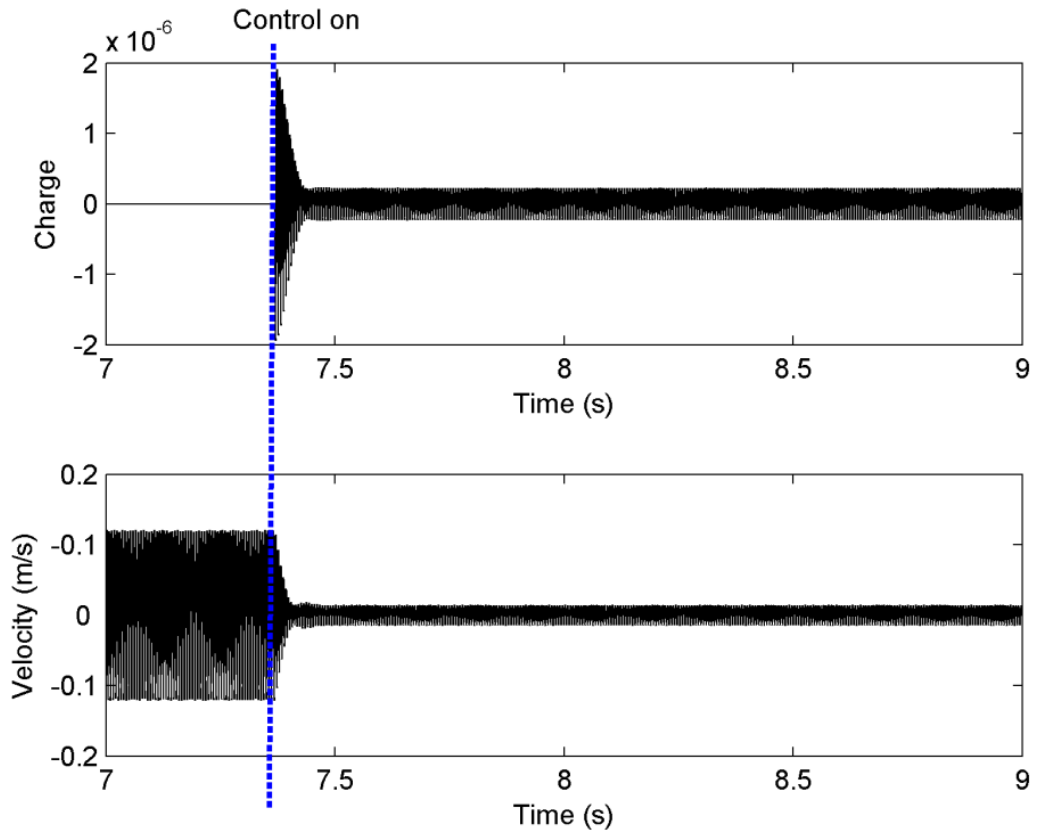

Fig. 6 Time response of $R L$ series shunt circuit. 

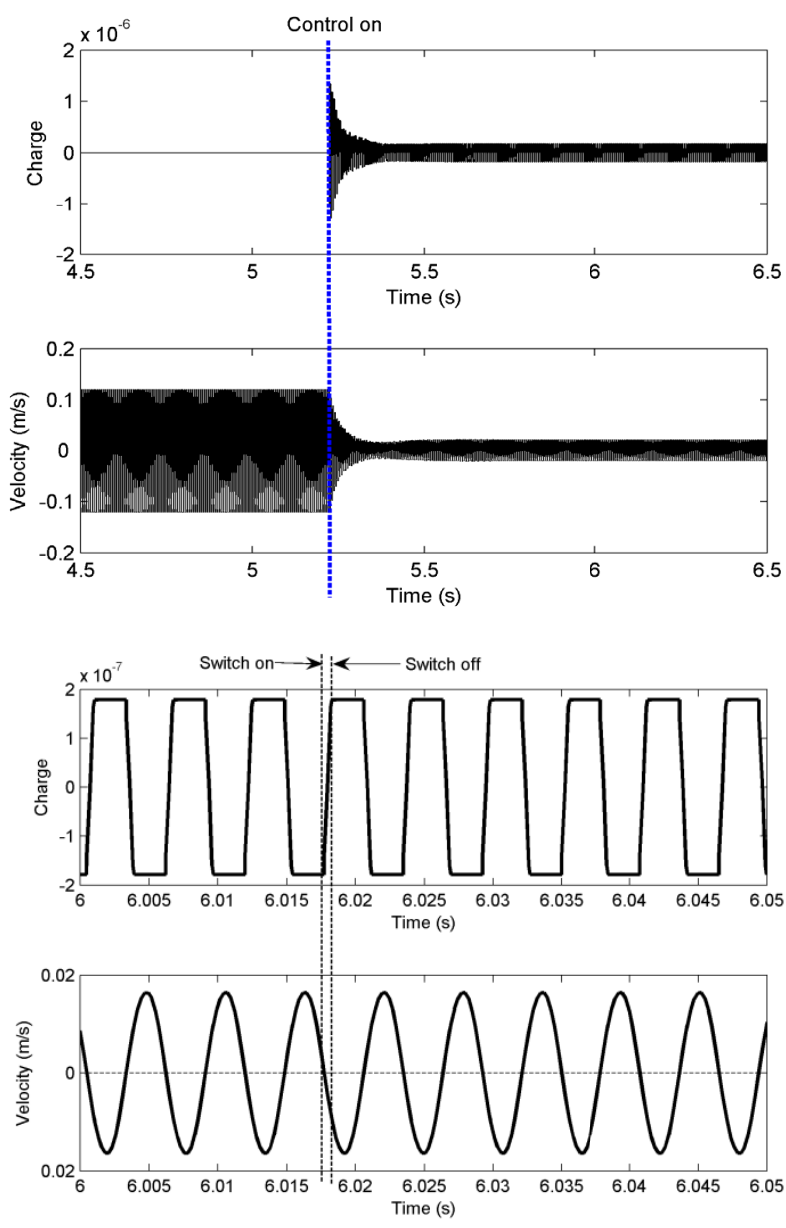

Fig. 7 Time response of pulse-switching shunt circuit.
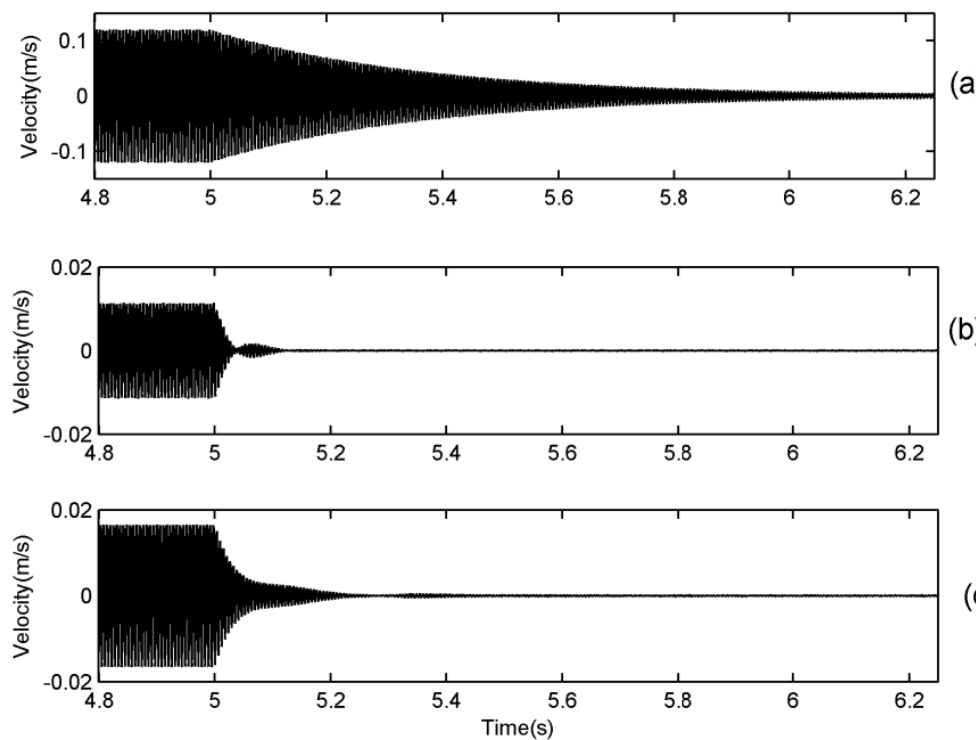

(c)

Fig. 8 Time response of the different shunt circuits (Sinusoidal burst finishing at $\mathrm{t}=5 \mathrm{~s}$ ). (a). Without control; (b). $R L$ series circuit; (c). Pulse-switching circuit. 
In the pulse-switching circuit, to obtain the maximum possible damping, the PZT actuator has to be connected to an $R L$ shunt circuit at exactly the moment when the modal velocity is zero. As the charge of the PZT actuator reaches a peak value that is opposite in sign to its initial charge, the switch must be open and the actuator charge remains constant until the switch closes again, as shown in Fig. 7. The applied charge is $180^{\circ}$ out of phase with modal velocity over each switch-open time. This is directly analogous to the well-known, direct velocity feedback control system (DVFB). The only difference between a pulse-switching circuit and DVFB is that for DVFB, the applied voltage varies over the half cycle, and for a pulse-switching circuit, the applied charge is constant over the half cycle. Fig. 9 compares the control performance between the $R L$ series and pulse-switching circuits. When the stiffness of a structure is changed, the performance of the pulse-switching circuit remains about $7 \mathrm{~dB}$. However, the performance of the $R L$ series circuit degrades quickly, the maximum sound power reduction is achieved only when the $R L$ series shunt circuit is precisely tuned to the required frequency of concern. This means the pulse-switching circuit is more stable than an $R L$ series circuit with regard to stiffness variations.

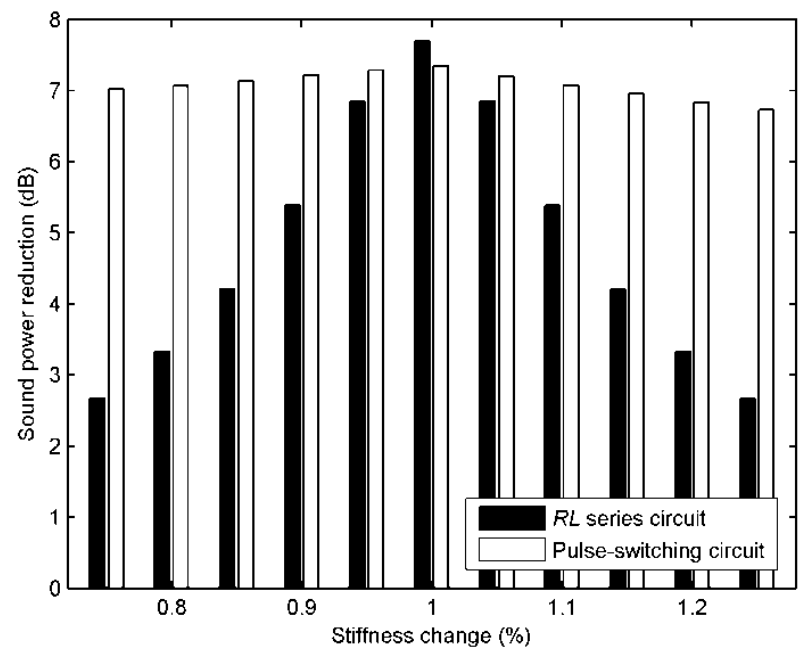

Fig. 9 Comparison of the control performance between an $R L$ series and a pulse-switching circuit.

\section{Experiment setup and results}

In order to further demonstrate the control performance of the $R L$ series, $R L$ parallel, $R L-C$ parallel and pulse-switching shunt techniques, an experimental test was performed on a clamped steel plate. This experiment is intended to demonstrate each of the four shunt circuits and to compare them in a real vibrating system. A $200 \mathrm{~mm} \times 200 \mathrm{~mm} \times 2 \mathrm{~mm}$ steel plate with two piezoelectric QP25W elements (One is used as the primary source and another is used for the shunt circuit), one piezo-fiber sensor which is used to drive switch controller, and one accelerometer bonded to its surface (center of plate) were used to monitor the control performance for each test. The plate was clamped at all boundaries. The test-setup is shown in Fig. 10. It should be noted that the switch controller was only used for the pulse-switching circuit. Fig. 11 shows a photograph of the clamped plate.

All frequency response functions (FRFs) were measured, from the voltage output of the accelerometer to the voltage input of the primary PZT actuator. A PULSE dynamic signal analysis system was used to create the excitation signal and perform all FRF measurements. The goal of the experiment was to control the radiated sound power due to the $(3,1)$ structural mode (with natural frequency $340 \mathrm{~Hz}$ ) of the plate by using different shunt 
circuits. Table 1 lists the values of the components used for each of the shunt circuits. All inductors used in the shunt circuits were passive inductors.

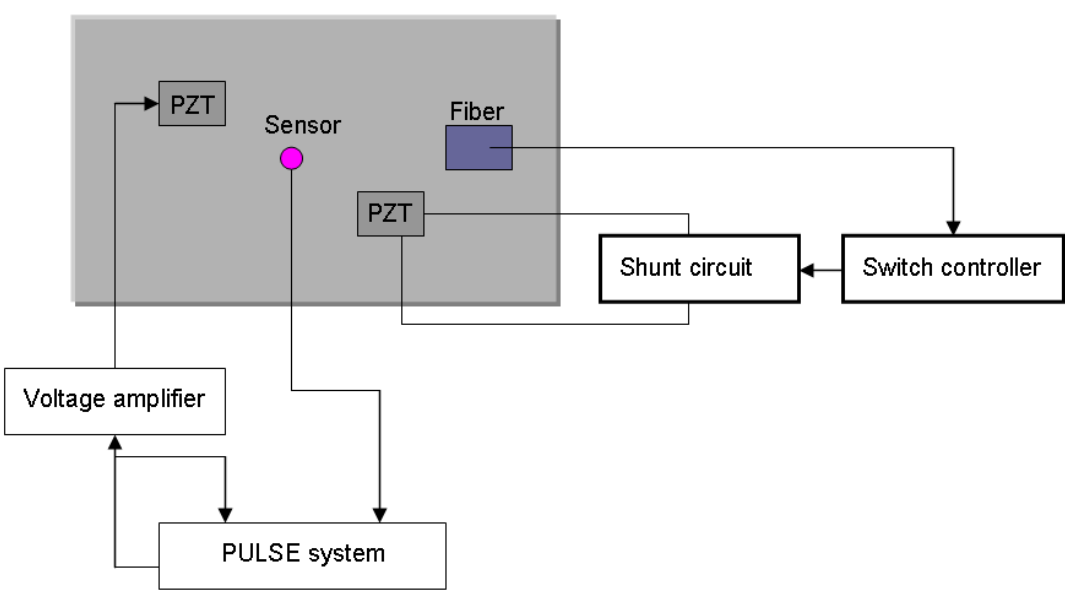

Fig. 10 Experiement setup.

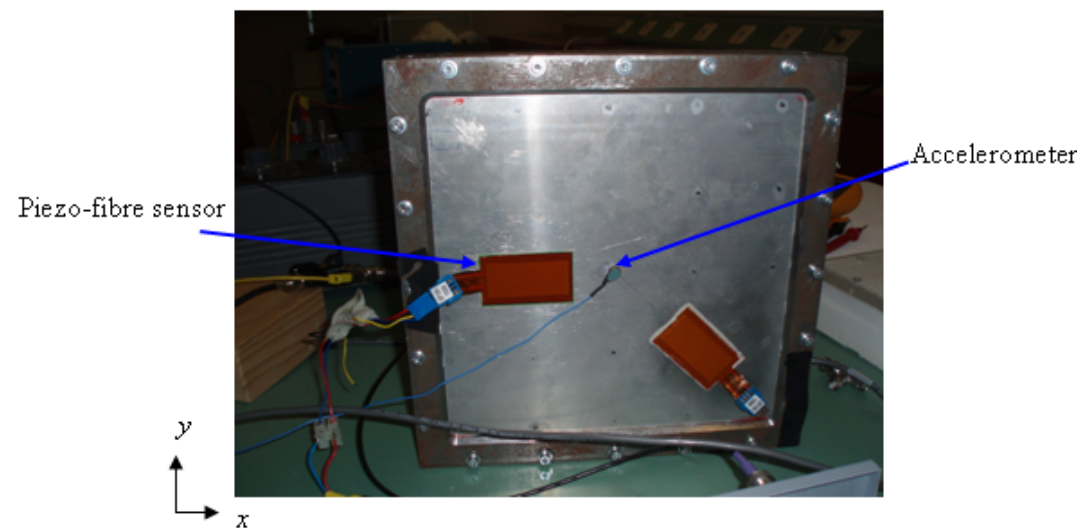

(a) Front of the plate

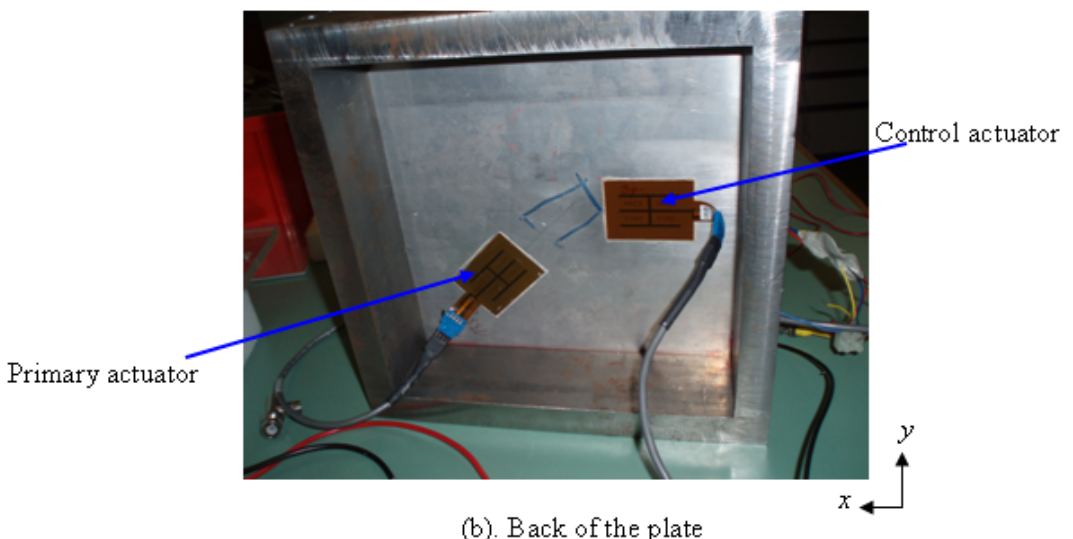

Fig. 11 The clamped plate used for the experimental validation.

In the first test, the control performance of the $R L$ series, $R L$ parallel and $R L-C$ parallel circuits were tested, and the results are shown in Figs. 12 and 13. Fig. 12 indicates that the shunt piezoelectric element can significantly reduce the resonant peak vibrations. Furthermore, the results also show that the control performances of the $R L$ series and $R L$ 
parallel circuit are basically the same. With $R L-C$ parallel circuits, the additional capacitance can reduce the value of inductance (See Table 1); however, the control performance has also reduced due to the additional capacitance, as shown in Fig. 13.

Table 1 Properties of shunt circuits used in experiments

\begin{tabular}{|c|c|c|c|c|c|c|}
\hline & \multirow{2}{*}{$R L$ series } & \multirow{2}{*}{$R L$ parallel } & \multicolumn{3}{|c|}{$R L-C$ parallel } & \multirow{2}{*}{ Pulse-switching } \\
\hline & & & Case A & Case B & Case C & \\
\hline Resistance $(\Omega)$ & 55 & $31 \mathrm{~K}$ & 35 & 28 & 10 & 10 \\
\hline Inductance $(\mathrm{H})$ & 0.71 & 0.72 & 0.54 & 0.445 & 0.318 & 0.071 \\
\hline Capacitance $(\mu \mathrm{F})$ & None & None & 0.1 & 0.2 & 0.4 & None \\
\hline
\end{tabular}

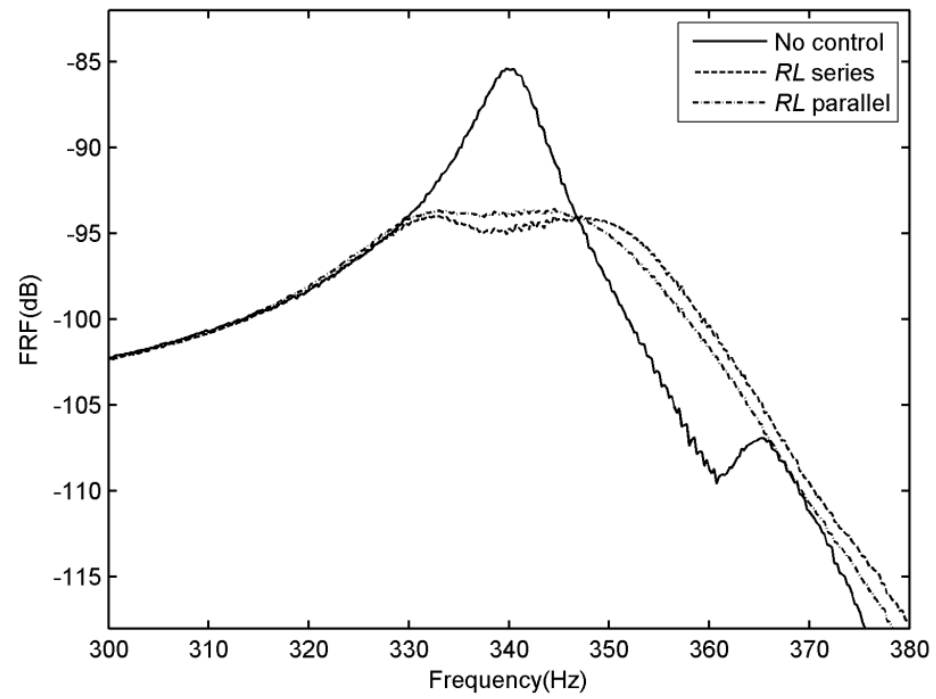

Fig. 12 Experimental comparison of $R L$ series and parallel shunt circuits.

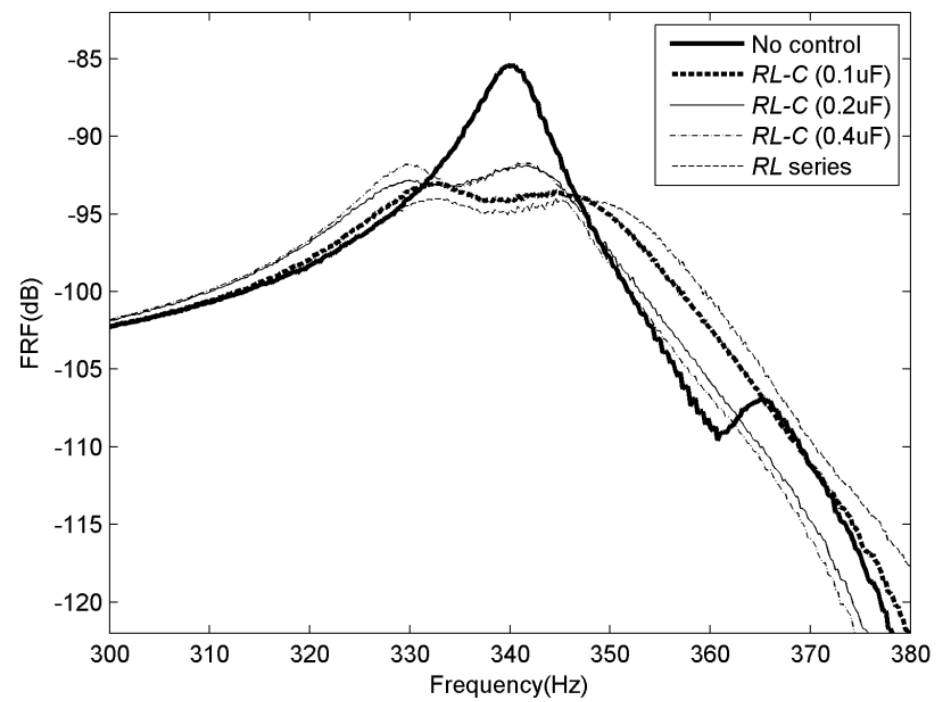

Fig. 13 Experimental comparison of $R L-C$ parallel shunt circuits for different capacitances.

Fig. 14 shows the experimental result using a pulse-switching circuit. It can be found that a pulse-switching circuit can achieve the same control performance as the $R L$ series 
circuit. By using a pulse-switching circuit, the value of the inductance can be reduced to $0.071 \mathrm{H}(10 \%$ of $R L$ series circuit). The advantages of the switching techniques are a small required shunt inductance, a lower sensitivity to environmental changes and easier tuning. Very low external power for the switch controller is required so it may be possible to extract this energy directly from the vibration of the structure itself.

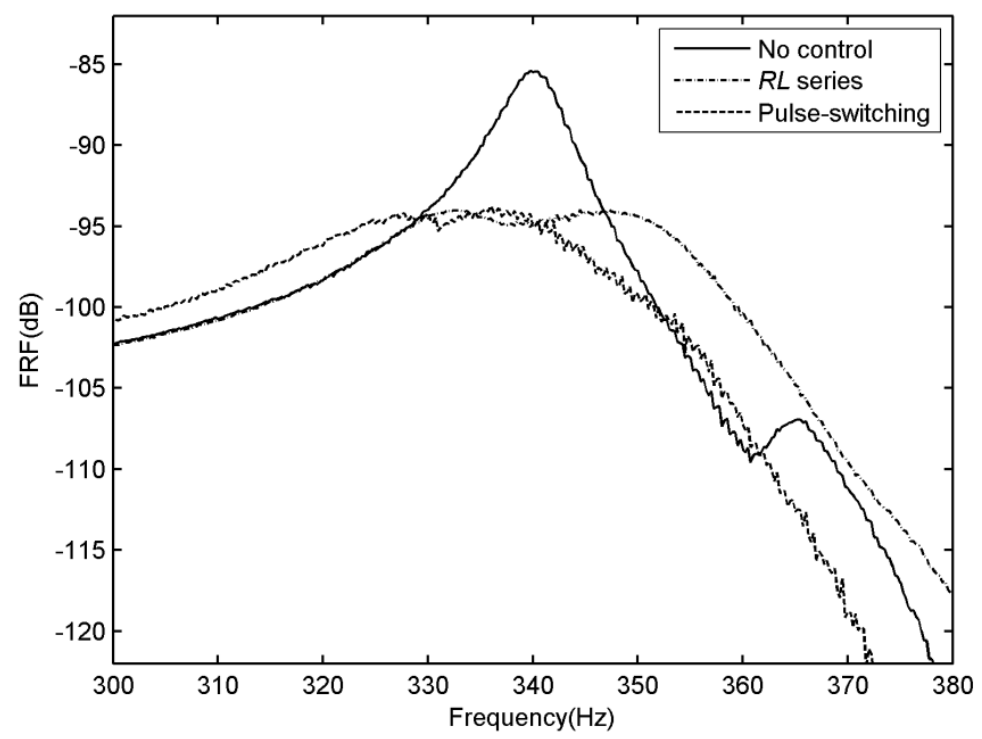

Fig. 14 Experimental comparison of $R L$ series and pulse-switching circuit.

The experiments conducted confirm that the shunt piezoelectric damping techniques presented in this paper are capable of effectively suppressing the vibration of the plate and the associated sound radiation. It was observed that for the target mode at $340 \mathrm{~Hz}$ (one of the strongly radiating modes, namely the $(3,1)$ structural mode) the $R L$ parallel, $R L$ series and pulse-switching circuits decrease the peak resonant amplitudes up to $9 \mathrm{~dB}$.

Since the shunt circuit is inherently passive and its control mechanism is based on adding damping to the target mode, the resonance response of the $(3,1)$ mode has been reduced over the entire plate thanks to the shunt circuit action. This means that the radiated sound power of the target mode is also reduced due to the lower vibration amplitude of the structure. It should be noted that the pulse-switching circuit can achieve a performance comparable to control with the traditional $R L$ series circuit.

\section{Conclusions}

In this study, several different shunt piezoelectric damping techniques, i.e. $R L$ series, $R L$ parallel, $R L-C$ parallel and pulse-switching shunt circuit, have been analyzed and compared. Using the impedance method, the piezoelectric shunt circuit can be seen as contributing additional frequency-dependence damping of the system. By minimization of the sound power of the structure, the optimal parameters for shunt circuits have been obtained. Also, the switch law for pulse-switching circuits has been discussed. Numerical simulations were performed for each of these shunts techniques, and it was found that the $R L$ series and $R L$ parallel circuit have basically the same control performance. Experimental results have been presented using a $R L$ series shunt circuit, a $R L-C$ parallel shunt circuit and pulse-switching circuit. The experimental results have shown that the vibration and radiated sound power of a structure can be reduced significantly by using these shunt circuits. The theoretical and experimental techniques presented in this study provide a valuable tool for effective shunt piezoelectric damping. 


\section{References}

[1] N. W. Hagood and A. von Flotow. Damping of structural vibration with piezoelectric materials and passive electrical networks. J. Sound Vib. 146, 243 - 268 (1991).

[2] C. H. Nguyen and S. Pietrzko. FE analysis of a PZT-actuated adaptive beam with vibration damping using a parallel R-L shunt circuit. Fin. Elem. Anal. Des. 42, 1231 - 1239 (2006).

[3] G. Caruso. A critical analysis of electric shunt circuits employed in piezoelectric passive vibration damping. Smart Mater. Struct. 10, 1059 - 1068 (2001).

[4] C. H. Park. Dynamics modelling of beams with shunted piezoelectric elements. $J$. Sound Vib. 268, 115-129 (2003).

[5] A. Agneni, F. Mastroddi and G.M. Polli. Shunted piezoelectric patches in elastic and aeroelastic vibrations. Computers and Structures. 81, 91-105 (2003).

[6] M. B. Ozer and T. J. Royston. Passively minimizing structural sound radiation using shunted piezoelectric materials. J. Acoust. Soc. Am. 114, 1934 - 1946 (2003).

[7] W. W. Clark. Vibration control with state-switching piezoelectric materials. J. Intell. Mater. Syst. Struct. 11, 263-271 (2000).

[8] L. R. Corr and W. W. Clark. Comparison of low-frequency piezoelectric switching shunt techniques for structures damping. Smart Mater. Struct. 11, 370 - 376 (2002).

[9] L. R. Corr and W. W. Clark. A novel semi-active multi-modal vibration control law for a piezoceramic actuator. Trans. ASME. J. Vib. Acoust. 125, 214 - 222 (2003)

[10] L. R. Corr and W. W. Clark. Energy dissipation analysis of piezoceramic semi-active vibration control. J. Intell. Mater. Syst. Struct. 12, 729 - 736 (2001).

[11] S. Pietrzko and Q. Mao. New results in active and passive control of sound transmission through double wall structures. Aer. Sci. Tech. 12, 42 - 53 (2008).

[12] D. Niederberger, S. Pietrzko and M. Morari. Noise control in a duct with online-tuned shunted piezoelectric materials. Proc. SPIE Smart Structures and Materials Conf.: Damping and Isolation, 5386, 405 - 413 (2004).

[13] Q. Mao, et al., A piezoelectric array for sensing radiation modes. Applied Acoustics. 64 (7), $669-680$ (2003).

[14] C. R. Fuller, P.A. Nelson and S. J. Elliott, Active control of vibration. Academic Press, London, 1996. 